\title{
The EUROnu neutrino oscillation super beam
}

\section{N. Vassilopoulos*, E. Baussan and M. Dracos}

IPHC, Université de Strasbourg, CNRS/IN2P3, Strasbourg F-67037, France

E-mail: nikolaos.vassilopoulosecnrs.iphe.fr

In the framework of the EUROnu design study, a new design for the CERN to Fréjus neutrino beam based on CERN Superconducting Proton Linac has been achieved. This super beam is able to discover a $\mathrm{CP}$ violation in the leptonic sector over a significant fraction of $\delta_{\mathrm{CP}}$ parameter by using a 4-MW proton beam of $4.5 \mathrm{GeV}$, a base-line of $130 \mathrm{~km}$ and the future Water Cherenkov MEMPHYS detector ( 0.50 Mton fiducial mass). The neutrino mass hierarchy could also be discoverd by combining atmospheric neutrino data. After discovering that the last neutrino oscillation angle $\theta_{13}$ was large, it came out that working on the second oscillation maximum is even more promising. Thus, using the same parameters than for CERN to Fréjus, CERN to Canfranc with a base-line of $650 \mathrm{~km}$ has even more physics potentialities. The technological challenges and the design of the target and horn as well as the safety issues due to the high irradiation produced inside the super beam apparatus are also discussed.

XV Workshop on Neutrino Telescopes,

11-15 March 2013

Venice, Italy

${ }^{*}$ Speaker. 


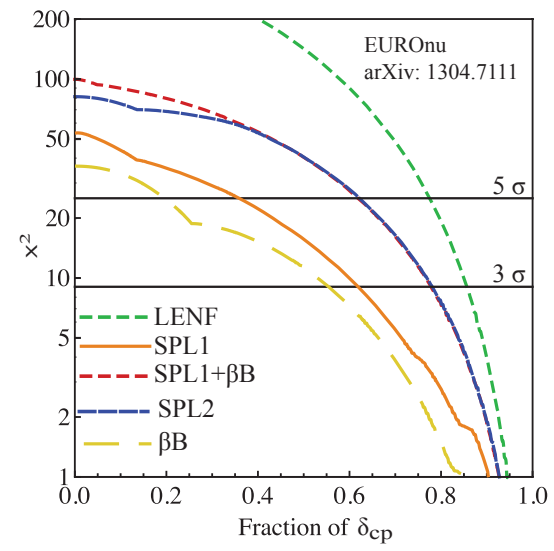

Figure 1: Significance of discovery as function of the fraction of the full $\delta_{\mathrm{CP}}$ space for SPL1, SPL2, SPL1 $\beta$ B and low energy neutrino factory LENF.

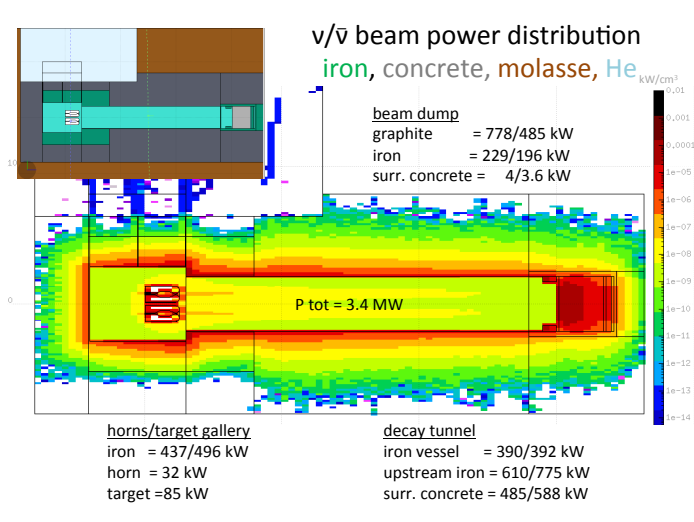

Figure 2: Power density distribution through-out the secondary beam line. A large portion of the $4 \mathrm{MW}$ power is dissipated in the four horns/targets station, shielding and beam dump.

\section{Precision measurements with the next generation of neutrino super beam}

\subsection{Super beam layout}

In the framework of EUROnu project [1] a new generation neutrino super beam has been studied [2] and is based on a relatively short base-line of $130 \mathrm{~km}$ from CERN to Fréjus underground laboratory in France where the megaton scale water Cherenkov detector MEMPHYS [3] could be installed. The neutrino source is expected to benefit from the Superconducting Proton Linac [4] at CERN that could produce a high intensity proton beam with $4 \mathrm{MW}$ power at $50 \mathrm{~Hz}$. This is a novelty since contemporary neutrino projects as T2K and NOvA are not expected to exceed $750 \mathrm{~kW}$ of power [5, 6] and also a technical challenge due to the extreme irradiation produced.

The proton beam line-up after the SPL linac includes an accumulator ring to reduce the proton pulse length in order to minimize the duration of the current pulses on the horn. The $4 \mathrm{MW}$ proton beam is distributed via four beam-lines onto a system of four horns/targets in order to minimize power dissipation and radiation problems. The beam is splitted by kicker magnets, then is bended by dipoles and finally is focused onto each target by a system of three quadrupoles [2].

The structure of the secondary beam facility is very compact based on the multi-mega watt four horns/targets station to produce/focus the charged mesons, a short decay tunnel $(25 \mathrm{~m})$ to produce the neutrino beam from the decaying focused particles, and a beam dump to stop the undecayed hadrons. Thick iron and/or concrete shielding is foreseen to confine the irradiation inside the apparatus. Special "radiation hot" cells are also included in the design allowing the replacement and manipulation of the high radioactive material with special equipment [2].

\subsection{Discovery potential}

The combined experiment of the super beam with MEMPHYS detector has a very good discovery potential of $\mathrm{CP}$ violation. The fraction of $\delta_{\mathrm{CP}}$ space that can be discovered in the Fréjus base-line (SPL1) is $62 \%$ and $37 \%$ at a significance level of $3 \sigma$ and $5 \sigma$ respectively. Better results 
could be achieved for the Canfranc base-line (SPL2) of $650 \mathrm{~km}$ whereas $80 \%$ and $60 \%$ at a significance level of $3 \sigma$ and $5 \sigma$ are covered respectively. For the Fréjus base-line the same level of performance could be reached only with the combined netrinos from a beta-beam [1] (SPL1+ $\beta$ B) as shown in figure 1. The neutrino mass hierarchy discovery significance level is lower than $3 \sigma$ and more than $5 \sigma$ for the SPL1 and SPL2 respectively [1]. In the case of SPL1, discovery is expected when data from the atmospheric neutrinos are combined as a benefit of a very large $\sin ^{2} 2 \theta_{13}$.

\section{Technological challenges}

Compared to the existing neutrino beams, the four horns/targets system has to be designed to work with a $4 \mathrm{MW}$ proton beam power and produce neutrinos at a $50 \mathrm{~Hz}$ rate. Many constraints appear due to the high particle rates and cycles that could limit the lifetime of the system. In the following subsections the target and horn designs are discussed as studied in the EUROnu project.

\subsection{Multi mega watt target technology}

A titanium alloy canister containing packed bed of titanium or beryllium spheres has been studied and found the best possible target candidate for the SPL neutrino beam [2]. This target has large surface area for heat transfer, coolant ability to access transversely areas with highest energy deposition, presents minimal stresses and offers potential heat removal rates at the hundreds kilowatt level. The canister is perforated with elliptical holes along its length used for cooling. Highly pressurized helium gas at 10 bar has been studied as coolant since it is almost beam neutral, presents low activation and no generation of stress wave, and do not create any corrosion problems. A 0.25 thick beryllium window with circumferential water cooling is foreseen as an interface between the proton beam and target station areas.

\subsection{Horn Studies}

A forward closed horn was studied [2] to be the best compromise between physics potentialities and reliability. The horn is made of aluminum $6061 \mathrm{~T} 6$ alloy because it presents a good trade off between mechanical strength, resistance to corrosion and electrical conductivity. Each horn has been studied under 1.3 MW proton beam power in case of one of the four horns fails to work. The thermodynamical studies were performed with a finite element model that allows the calculation of the horn stress and deformation due to the magnetic pressure and thermal dilatation. As result a low relatively stress under $30 \mathrm{MPa}$ is achieved when the horn has a uniform temperature of $60^{\circ} \mathrm{C}$. To maintain a constant working temperature in the conductors of the horn, water jets have been proposed for cooling. Preliminary fatigue studies indicate that each horn can withstand a year $\left(10^{8}\right.$ pulses) of operation.

In the power supply design [7], a one-half sinusoid current waveform with a $350 \mathrm{kA}$ maximum current and pulse length of $100 \mu \mathrm{s}$ at $50 \mathrm{~Hz}$ frequency is generated and distributed to four-horns. A bench of capacitors is charge and then discharged to each horn via a set of strip-lines. A current recovery stage allows a high energy recovery efficiency of $97 \%$ and thus it limits the power consumption. For feasibility reasons, a modular architecture has been adopted with 8 modules connected in parallel to deliver $44 \mathrm{kA}$ peak currents into the four-horn system. 


\subsection{Safety and activation studies}

The SPL super beam design has to take into account the significant amount of the produced radiation and material activation. The shielding should reduce the dose equivalent rate for workers to a minimal level. In order to reach these dosimetry objectives the ALARA (As Low As Reasonably Achievable) approach has already been used in the safety design. It consists of an iterative process between three phases: a) preparation, design of the facility, dose equivalent rate map and intervention procedures for workers b) execution, engineering phase check/improve the dosimetry objectives and c) feedback on safety from others experiments. In each of the three phases, the individual/collective intervention scenario for workers will be elaborated/modified to reduce external exposition to radiation to the minimum level. Several configurations of iron thicknesses surrounded by concrete have been investigated to reduce the dose equivalent rate to an acceptable level around the target station and minimize rock activation during beam operation [2]. The power density distribution along the secondary beam-line is shown in figure 2.

\section{Conclusions}

The SPL neutrino super beam has a great physics potentiality to discover the CP violation in the neutrino sector as well as the neutrino mass hierarchy. Studies performed during the EUROnu project show that the target and horn could be able to operate under extreme irradiation conditions. Future R\&D programs are needed to build and test the horn/target designs, the cooling methods of the beam elements and the power supply unit.

\section{Acknowledgments}

The authors would like to thank their collaborators in the EUROnu project for making these results possible.

\section{References}

[1] T. R. Edgecock et al., The EUROnu Project, Phys. Rev. ST Accel. Beams 16 (2013) 021002 [arXiv:1305.4067 [physics.acc-ph]].

[2] E. Baussan et al., The SPL-based Neutrino Super Beam, arXiv: 1212.0732 [physics.acc-ph].

[3] L. Agostino et al., Future large-scale water-Cherenkov detector, Phys. Rev. ST Accel. Beams 16 (2013) 061001 [arXiv:1306.6865 [physics.ins-det]].

[4] Conceptual design of the SPL II, a high-power superconducting H-linac at CERN, CERN-2006-006.

[5] T. Ishida, T2HK: J-PARC upgrade plan for future and beyond T2K, arXiv: 1311.5287 [hep-ex].

[6] M. Muether, NOvA: Current Status and Future Reach, Nucl. Phys. Proc. Suppl. 237-238 (2013) 135.

[7] E. Baussan et al., Study of the pulse power supply unit for the four-horn system of the CERN to Fréjus neutrino super beam, JINST 8 (2013) T07006 [arXiv:1304.7111 [physics.acc-ph] ]. 